\section{Imaging Gas-Solid Interactions in an Atomic Resolution Environmental TEM}

Xiao Feng Zhang* and Takeo Kamino ${ }^{* *}$

${ }^{*}$ Hitachi High Technologies America, Pleasanton, CA

${ }^{* *}$ Hitachi High Technologies Corp., Hitachinaka, Ibaraki, Japan

Xiao.zhang@hitachi-hta.com

\section{Introduction}

It is well known that analysis using transmission electron microscopes (TEM) yields very high resolution images of thin specimens. However, the applicability of TEM analysis is not universal due to the requirement that a high internal vacuum is required. This high vacuum precludes the TEM study of living specimens or specimens in a gas or liquid environment. In order to tackle this problem, L. Marton of Universite Libre in Brussels, Belgium was the first to design an environmental cell (E-cell) in 1935 that was sealed in the tip of a TEM sample holder [1]. Marton's design included two $0.5 \mu \mathrm{m}$ aluminum foils as upper and lower windows sandwiching a biological sample to sustain a living environment. The electron transparent windows permitted the confined biological objects to be imaged in TEM mode. Since then, environmental TEM (E-TEM) has received increasing attention from biological scientists and eventually from materials scientists as well.

\section{How is E-TEM realized?}

The purpose of the E-TEM is to introduce a gas or liquid atmosphere into the sample chamber of a TEM to study the interactions between the sample and gas or solvent. Since TEMs work at high vacuum, the introduction of gas or solvent in the sample area is realized by one of two ways in today's E-TEMs.

The first method is to seal the sample in an E-cell confined by electron transparent windows similar to the one Marton invented in 1935. The window materials must be nonporous and sufficiently strong to accommodate liquid or gas pressure, and should not be degraded by the cell environment or by the instrument's vacuum or the electron beam. The cell windows should not produce electron diffraction, so as not to interfere with electron diffraction from the sample. Current practice is to make the windows from amorphous carbon or amorphous silica, a couple of tens of nanometers thick. The advantages of this window type are that the E-cells have a simple design and a low cost because no modification of the TEM is required. This cell type is good for working with both gas and liquid environments, and the gas pressure can be as high as 300 torr (half atmosphere). Large-angle electron diffraction is obtainable. However, the drawbacks are also obvious. The total thickness of the sample, windows, and the gas path deteriorates the image resolution, therefore atomic resolution becomes very difficult to achieve if not impossible. Sample tilting makes the situation even worse. This is the main reason for using ultrahigh voltage TEMs, $(1 \mathrm{MeV}$ or higher) for E-TEM studies in the early years in an attempt to benefit from the increased penetration power and higher spatial resolution attendant to higher accelerating voltage instruments. However, the damage to the sample by the high energy electron beam, and the high price and inconvenient operation and maintenance service needed limited this approach's popularity. Sample exchange is troublesome and the sample may translate within the cell during analysis causing problems. Heating the sample in the microscope is another big concern because window materials may be damaged and broken at elevated temperatures.
The second method provides for a gas environment around the sample without a confined cell. Specially designed differential pumping systems are added to the microscope. In this system, two (or more) pairs of restricting apertures, above and below the sample area, replace the nonporous windows in the window type E-cells to partially pressure-isolate the increased pressure in the sample area from the high-vacuum regions above and below the sample. The gas introduced into the sample area will first leak through the inner pairs of restricting apertures, usually $0.1 \mathrm{~mm}$ in diameter and most of the gas volume will be pumped away by a liquid nitrogen cold trap and a mechanical pump connected to this intermediate zone. The gas leak rate through an aperture is proportional to the aperture diameter squared and the gas pressure. What is left of the gas passes through the outer pairs of restriction apertures, 0.2 to $0.4 \mathrm{~mm}$ in diameter, and is pumped out by the microscope vacuum system. This type of two-compartment, differential pumping E-cell concept assures that no severe change in TEM column vacuum occurs when gas is admitted into the sample area, something like the multi-compartment design in submarines and steamships to prevent or slow down significant water leakage in an impact accident.

Perhaps the biggest advantage of the differential pumping Ecells is that the TEM sample is now directly exposed to the electron beam, therefore high resolution imaging, sample tilting, and in-situ heating, that are concerns with window type E-cells, are not problems anymore. Easy operation is another advantage, once such a robust differentially pumped E-cell is installed, large numbers of specimens can be studied. The constant effusion of gas from the restricting apertures also helps to prevent contamination of the specimen. However, adding both restricting apertures in the optical path and the associated additional pumping system turns the microscope into a dedicated E-TEM. Design, manufacture, and installation of the instrument is more complicated and requires much longer time. The price and maintenance cost of the machine can be doubled compared to conventional TEMs. The restricting apertures below the sample also limit the collectable diffraction angle, therefore, high-angle electron diffraction phenomena can not be recorded. Also, because the sustainable pressure differential across a sealed single aperture of regular size is limited to a factor of $\leq 100 \times$, the maximum gas pressure in the sample area is generally limited to less than 100 Torr to maintain the required high vacuum in rest of the TEM column.

\section{Atomic resolution - A new capability for gas/heating E- TEM}

In materials science and industry, gas-solid interactions are among the most fundamental physical and chemical objects of study as they control the catalysis, the fuel cell process, the materials and nanomaterials synthesis and growth processes, the phase transformation process, and the gas sensing and detecting processes. Traditionally, gas-solid interactions can be studied by analyzing the structural phases before and after reactions. Knowledge obtained in this way is macroscopic and can be expressed in phase diagrams and chemical equations. Understanding of the time-resolved intermediate stages in reactions has been a challenge. With the advent of nanoscience and nanotechnology, materials of interest in many gas-solid interaction studies shrink to the nanometer scale, and knowledge of the time- and temperature-resolved dynamic and kinetic mechanisms on the atomic level is demanded. It turns out that in-situ heating high-resolution electron microscopy in gas 


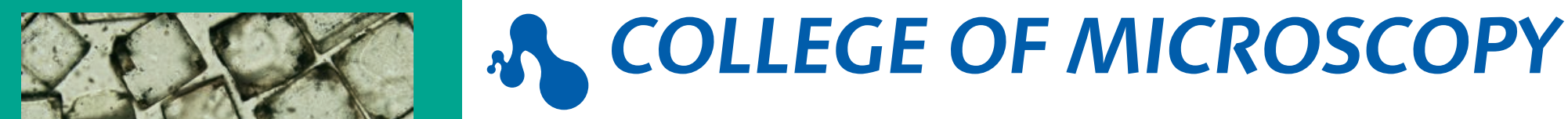

Located in Westmont, Illinois, the College of Microscopy is an institution for specialized instruction and education, whose goal is to advance the knowledge and understanding of light and electron microscopy for materials analysis.

One of the advantages of attending a course at the College of Microscopy is that our courses are taught primarily by experienced senior staff scientists from

McCrone Associates, Inc. who solve micro-analytical problems on a daily basis. That's why it's true when we say,

\section{"Learn From Experience...}

\section{Learn From The Experts."}

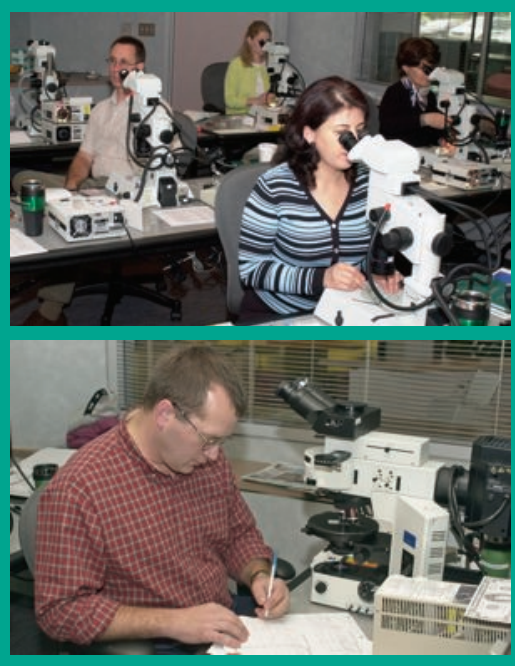

\section{Polarized Light Microscopy}

COM100: Polarized Light and Chemical Microscopy

Oct. 16-20, 2006

COM160: Techniques of Optical Crystallography July 16-20, 2007

\section{Electron Microscopy}

COM200: Scanning Electron Microscopy

Oct. $16-20,2006$

COM250: Transmission Electron Microscopy

Oct. 31-Nov. 2, 2006

\section{Imaging/Software}

COM500: Image Analysis Workshop Taught by John Russ, Ph.D.

Sept. 18-20, 2007

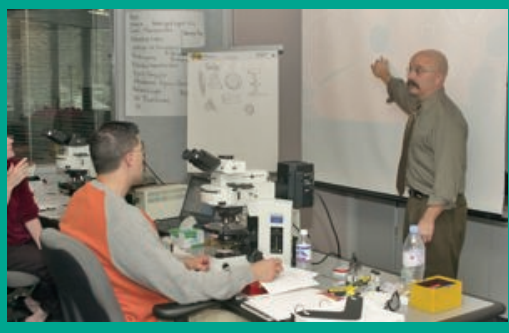

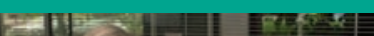

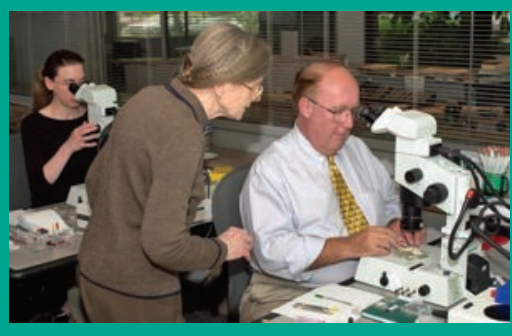

COM510: Scientific Imaging

Nov. 13-17, 2006

\section{Sample Preparation}

COM300: Microscopic Particle Handling

Oct. 2-6, 2006

\section{Special Applications}

COM400: Identification of Opaque Minerals

Aug. 13-15, 2007

COM410: Universal Stage Methods

Aug. 16-17, 2007

COM430: Microscopical Identification of White-

Nov. 13-17, 2006

Powder Unknowns (Refresher)

\section{Spectroscopy}

COM600: Infrared Microscopy

Nov. 7-9, 2006

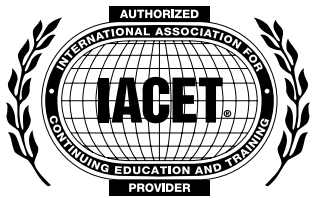

IACET CEUs awarded for all courses completed with the College of Microscopy

College of Microscopy $\bullet 850$ Pasquinelli Drive, Westmont, Illinois 60559 www.collegeofmicroscopy.com 


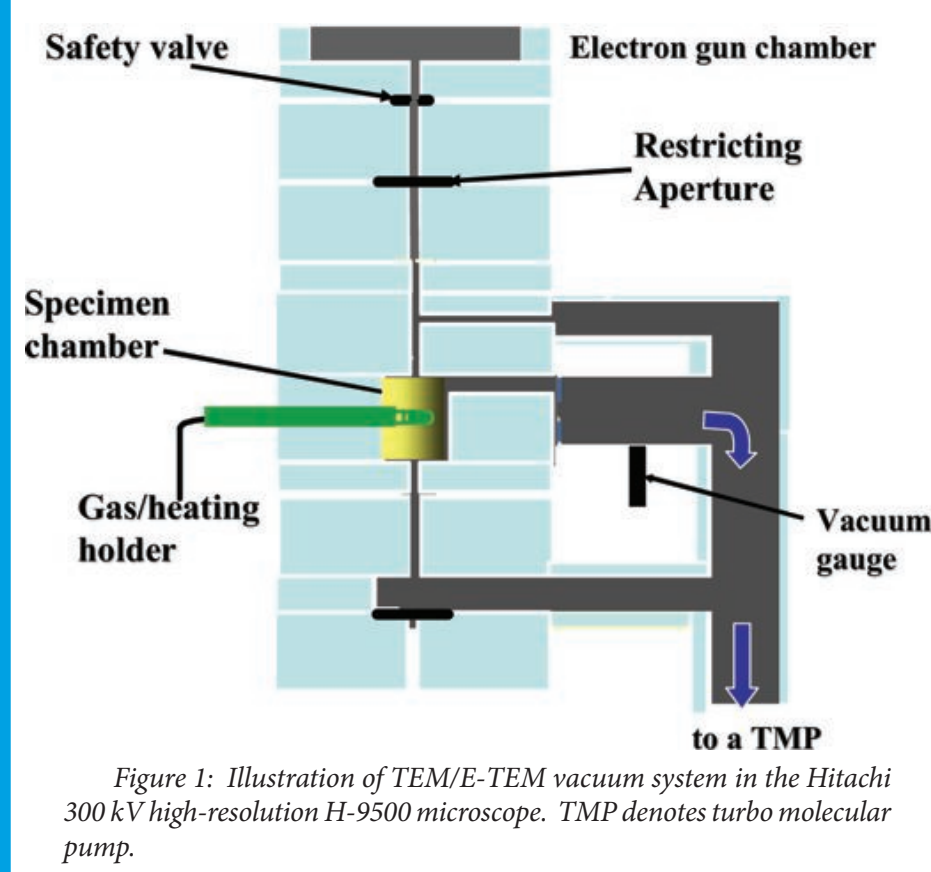

environments becomes indispensable for analyzing nanoscale and often powder-like materials. The automobile industry, government laboratories, and universities are investing millions of dollars in building complicated in-situ gas-heating E-TEMs with atomic resolution capability.

\section{Affordable, high-resolution TEM/E-TEM technology}

Hitachi is introducing an innovative TEM/E-TEM design. The $300 \mathrm{kV}$ high resolution H-9500 TEM, which has a unique design for its vacuum system as schematically illustrated in Fig. 1.

As can be seen in Fig. 1, compared with the traditional TEM vacuum system, a high speed turbo molecular pump is added to pump the specimen chamber, a $0.3 \mathrm{~mm}$ restricting aperture above the specimen chamber is installed to restrict the gas leakage into the upper column, and a safety valve in the gun area is used to further maintain the safe high vacuum in the gun area. The whole system is indeed a sort of built-in, simplified differential pumping system that allows gas environmental TEM studies without having to modify the entire TEM. The instrument is a fully-functional high resolution TEM with full tilting and high-angle diffraction recording ability, but when needed, the gas injection/heating capability can be introduced in conjunction with a Hitachi patented windowless gas/heating holder pioneered and developed by T. Kamino [2,3]. The holder requires no self-confined window cell, and can heat the sample to above $1000^{\circ} \mathrm{C}$ with the injection of gases like $\mathrm{H}_{2}, \mathrm{O}_{2}$, air, water vapor, and others.

To capture gas-solid interactions at atomic resolution, the gas pressure in the sample area must be carefully set. The maximum gas pressure achievable in the sample area of the H-9500 TEM is around $0.1 \mathrm{~Pa}\left(\sim 10^{-3}\right.$ torr $)$. This is not a high pressure compared with the maximum pressure achievable in the window type E-cell or even in the dedicated differential pumping E-TEM. However, this level of pressure and the stable gas/heating sample stage guarantee that atomic resolution imaging for dynamic structural evolution is possible. Too high a pressure will not only degrade the image resolution due to the strong inelastic scattering of the incident electron by the gas molecules, but also the resulting gas-solid interactions are often too fast to be recorded even by a high speed video camera. To further eliminate the effect of the inelastic electron scattering, an imaging filter can be attached to the microscope. Chemical analysis is performed using energy-dispersive X-ray spectroscopy (EDX) or electron energy loss spectroscopy (EELS).

A major concern in practical in-situ heating electron $\mathrm{mi}-$ croscopy experiments is how to keep the specimen stable and in focus during high

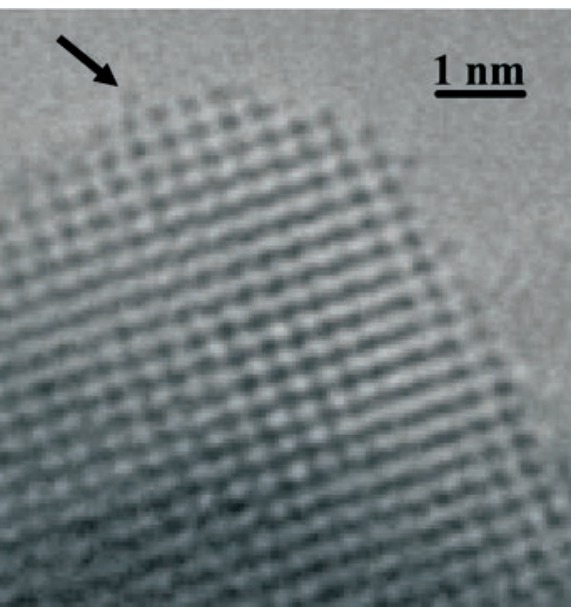

Figure 2: Atomic resolution study of $\mathrm{SnO}_{2}$ crystal growth at $200^{\circ} \mathrm{C}$ in a $2 \times 10^{-2} \mathrm{~Pa}$ in air. The arrow indicates a single atom on the growth surface. resolution observation at elevated temperatures. The problem is caused by the drift of the specimen along with the metal grid, which serves as a support for the specimen, suspended in the furnace type heating chamber. Changing temperatures or temperature fluctuations caused by gas injection introduces thermal expansion or contraction of the metal grid, resulting in a quite considerable specimen drift. The drift can easily last for an hour although piezocontrolled anti-drift system may help to shorten the time. To get around this problem, Kamino et al. developed the Hitachi heating and gas-heating stages with an innovative design. In Hitachi holders, the traditional furnace type heating chamber is replaced by helically-wound tungsten heating filaments that have direct contact with the specimen, therefore no metal grids are needed $[2,3]$. The drift rate is largely minimized, focus is easy to maintain, and the waiting time for consistent focus and imaging-ready conditions is only about $10 \mathrm{~min}$.

Fig. 2 shows an application example using the Hitachi TEM/ETEM technology. The high resolution image was taken at $200^{\circ} \mathrm{C}$ to show the growth of a $\mathrm{SnO}_{2}$ crystal in air with a pressure of $2 \times$ $10^{-2} \mathrm{~Pa}\left(1.5 \times 10^{-4} \mathrm{Torr}\right)$. The dark contrast spots correspond to the projected atomic columns.

It can be expected that with the increased interest of the nanoscience and nanotechnology communities, as well as the renewable energy development community, demand for affordable yet high performance atomic resolution TEM/E-TEM system will be huge. In particular, the automobile industry, fuel industry, chemical industry, nanomaterials industry, sensor industry, and large research facility centers in academia must have this atomic resolution, easy operation, high sample throughput, robust TEM with E-TEM capability to lead the competition and make innovations.

\section{References:}

1. L. Marton, "Electron Microscopy of Biological Objects", Nature 133 (1934) 911.

2. T. Kamino, and H. Saka, "Newly developed high resolution hot stage and its application to materials science", Microsc. Microanal. Microstruct. 4 (1993) 127-135

3. T. Kamino, T. Yaguchi, M. Konno, A. Watabe, T. Marukawa, T. Mima, K. Kuroda, H. Saka, S. Arai, H. Makino, Y. Suzuki and K. Kishita, "Development of a gas injection/specimen heating holder for use with transmission electron microscope", J. of Electron Microscopy 54 (2005) 497. 


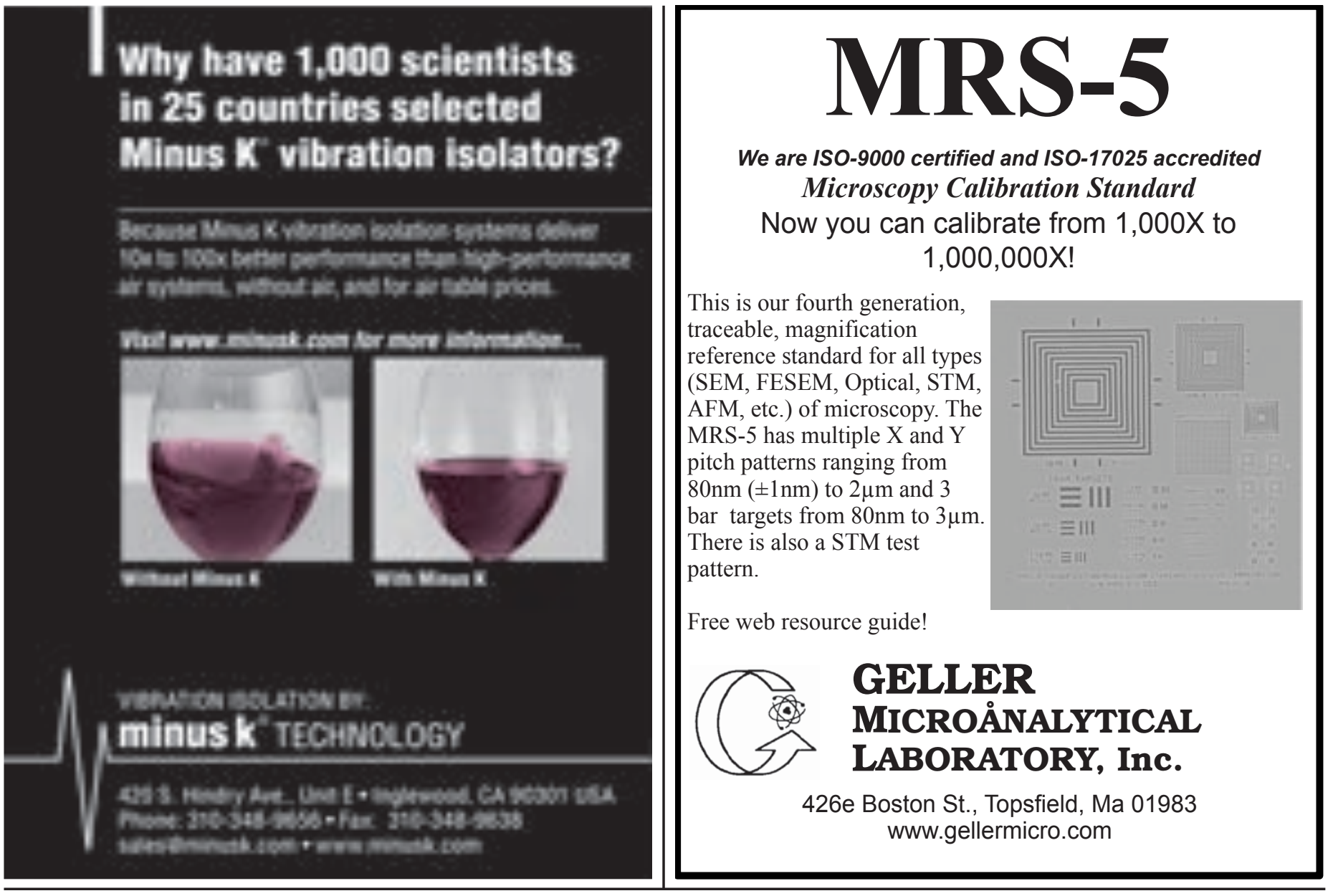

e2v scientific instruments see what we're made of

\section{Introducing the SiriusSD range - setting a new standard in high performance liquid nitrogen $\left(\mathrm{LN}_{2}\right)$-free $\mathrm{X}$-ray detection}

SiriusSD is a silicon drift detector designed to make short work of X-ray analysis. Its industry-standard restored preamplifier output provides some compelling benefits. e2v scientific instruments is the only independent EDX detector company offering true worldwide support, with repair and upgrade facilities based in both Europe and the USA.

\section{SiriusSD range}

- High rate capability

- Excellent resolution

- Stable peak position and resolution over a broad range of count rates

- Flexible system integration

- Available in both EDX and XRF cryostat formats

To find out more about our products and new facilities, please visit: www.e2vsi.com e2v scientific instruments was previously known as Gresham
UK:

T: +44 (o) 1628533060 E: e2vsi@ezv.com
USA:

T: +1 914592 6050, Ext 892

E: e2vsi-na@e2v.com see what we're made of 